\title{
Stereotactic radiosurgery and immunotherapy for metastatic spinal melanoma
}

\author{
James P. Caruso, BS, ${ }^{1}$ Or Cohen-Inbar, MD, PhD, ${ }^{1,7}$ Mark H. Bilsky, MD, ${ }^{2,3}$ \\ Peter C. Gerszten, MD, MPH, ${ }^{4,5}$ and Jason P. Sheehan, MD, PhD ${ }^{1,6}$ \\ Departments of ${ }^{1}$ Neurological Surgery and ${ }^{6}$ Radiation Oncology, University of Virginia Health System, Charlottesville, Virginia; \\ 2Department of Neurosurgery, Memorial Sloan-Kettering Cancer Center; ${ }^{3}$ Department of Neurological Surgery, Weill Cornell \\ Medical College, New York, New York; Departments of ${ }^{4}$ Neurological Surgery and ${ }^{5}$ Radiation Oncology, University of Pittsburgh \\ School of Medicine, UPMC Health System, Pittsburgh, Pennsylvania; and ${ }^{7}$ Molecular Immunology and Tumor Immunotherapy \\ Laboratory, Technion-Israel Institute of Technology, Haifa, Israel
}

\begin{abstract}
The management of metastatic spinal melanoma involves maximizing local control, preventing recurrence, and minimizing treatment-associated toxicity and spinal cord damage. Additionally, therapeutic measures should promote mechanical stability, facilitate rehabilitation, and promote quality of life. These objectives prove difficult to achieve given melanoma's elusive nature, radioresistant and chemoresistant histology, vascular character, and tendency for rapid and early metastasis. Different therapeutic modalities exist for metastatic spinal melanoma treatment, including resection (definitive, debulking, or stabilization procedures), stereotactic radiosurgery, and immunotherapeutic techniques, but no single treatment modality has proven fully effective. The authors present a conceptual overview and critique of these techniques, assessing their effectiveness, separately and combined, in the treatment of metastatic spinal melanoma. They provide an up-to-date guide for multidisciplinary treatment strategies. Protocols that incorporate specific, goaldefined surgery, immunotherapy, and stereotactic radiosurgery would be beneficial in efforts to maximize local control and minimize toxicity.
\end{abstract}

http://thejns.org/doi/abs/10.3171/2014.11.FOCUS14716

KEY WORDS metastatic spinal melanoma; stereotactic radiosurgery; separation surgery; immunotherapy; review

$\mathrm{M}$ ETASTATIC melanoma poses an onerous clinical burden. Its incidence is rising rapidly in the US and abroad,$^{38}$ and the median survival time of patients with advanced melanoma is less than 10 months. ${ }^{26}$ This trend derives from myriad factors that render melanoma difficult to treat. Melanoma has demonstrated a propensity for early metastasis, ${ }^{6}$ and its vascular characteristics amplify the difficulty of surgical excision. ${ }^{29,42}$ Both of these factors contribute to the high recurrence rates observed following resection. ${ }^{5}$ Additionally, data demonstrate that metastatic melanoma is relatively resistant to both conventional external-beam radiation therapy (cEBRT) ${ }^{13,14,28,47,49}$ and chemotherapy. ${ }^{49,92}$ Finally, melanoma is capable of downregulating proliferation pathway gene expression to assume a more invasive phenotype.$^{55}$ This property, known as dynamic phenotype switching, enables melanoma to metastasize rapidly in response to specific environmental stimuli, such as hypoxia. This adaptation complicates treatment decisions because its onset is difficult to predict. Dynamic phenotype switching, a feature of highly malignant neoplasms, allows the tumor cells to alternate between proliferative and invasive phenotypes in response to external challenges (such as hypoxia, radiation, or chemotherapy), contributing to its ability to resist chemotherapy and radiation therapy. Additionally, hypoxia-induced alterations in tumor cell genotype can influence response to immunotherapeutic techniques, including BRAF and ERK inhibitors.

ABBREVIATIONS cEBRT = conventional external-beam radiation therapy; CTLA-4 = cytotoxic T-lymphocyte antigen-4; IFN = interferon; IL-2 = interleukin-2; LINAC = linear accelerator; $\mathrm{MHC}=$ major histocompatibility complex; NK = natural killer; NOMS = Neurological deficits, Oncological features, Mechanical spinal instability, and Systemic disease progression; PD-1 = programmed death-1; PD-L1, -L2 = programmed death-1 ligands 1 and 2; SRS = stereotactic radiosurgery; Th1 = helper T cell Type 1; TIL = tumor-infiltrating lymphocyte.

SUBMITTED October 21, 2014. ACCEPTED November 24, 2014

INCLUDE WHEN CITING DOI: 10.3171/2014.11.FOCUS14716.

DISCLOSURE The authors report no conflict of interest concerning the materials or methods used in this study or the findings specified in this paper. 
When metastatic melanoma presents in the spine, these obstacles become more prominent. The spinal cord can tolerate maximal, single-fraction radiation doses of approximately 14 Gy to a point dose on the spinal cord (cord Dmax $)^{94}$ which is substantially lower than the tolerance of surrounding normal tissue. Considering melanoma's radioresistant histology and the spinal cord's increased susceptibility to radiation damage, cEBRT lacks the precision required to deliver a dose that is both safe and therapeutic, whereas radiosurgery offers the ability to deliver a higher therapeutic dose with the precision necessary to spare adjacent critical structures. ${ }^{28}$ Surgical excision of metastases also requires great caution and conveys operative risks and rehabilitation needs for patients who already have systemic disease. These treatment limitations prove particularly relevant, considering that the incidence of melanoma has substantially increased over the past 40 years ${ }^{67}$ and the spine is the most common site for melanoma metastases. ${ }^{29,81}$

This review is designed to evaluate the efficacy of surgical therapy, radiosurgery, and immunotherapy for metastatic spinal melanoma, separately and in combination. For the purpose of this review, we focus our analysis on treatment of extramedullary paraspinal melanoma metastases. In our experience, intramedullary metastases, or metastases that demonstrate leptomeningeal spread, are not suitable candidates for traditional surgical or radiosurgical intervention. We provide a conceptual overview of recent developments in melanoma immunotherapy, and we devote particular attention to the application of the concept of "separation surgery" and stereotactic radiosurgery (SRS) to treat extramedullary metastatic spinal melanoma.

\section{Immunotherapy}

\section{Introduction to Melanoma Immunotherapy}

The FDA approvals of interleukin-2 (IL-2) in 1998 and ipilimumab in 2011 were major breakthroughs in the development of immunotherapy for melanoma. ${ }^{78}$ These treatments exemplify 2 important classifications of immunotherapy, cytokine based and checkpoint blockade based. Although checkpoint blockade-based therapy demonstrates improved tumor control and reduced toxicity compared with cytokine-based therapy, clinical trials are still being conducted to evaluate the efficacy of both treatments. Cytokine-based therapy involves administration of recombinant cytokines to potentiate the activity of endogenous immune cells and to drive immune differentiation toward the helper T cell Type 1 (Th1) cytotoxic subgroup. Checkpoint blockade-based therapy impairs regulatory pathways to amplify the immune response to tumors. Table 1 summarizes important studies for each of these 2 classes of immunotherapy.

Although we have limited our focus to the above strategies, it is worth noting that specific targeted antibody therapies also show great promise. ${ }^{78} \mathrm{~A}$ popular subject of targeted therapy is BRAF, a serine/threonine kinase that upregulates MEK kinase activity in the Ras-Raf-MEK-ERK pathway. Melanoma cells with BRAF V600E mutations have a worse prognosis than melanoma cells with wild-
TABLE 1. Studies of immunotherapy for metastatic melanoma

\begin{tabular}{llcc}
\hline \multicolumn{1}{c}{ Authors \& Year } & \multicolumn{1}{c}{$\begin{array}{c}\text { Mechanism of } \\
\text { Immunotherapy }\end{array}$} & $\begin{array}{c}\text { No. of } \\
\text { Patients }\end{array}$ & $\begin{array}{c}\text { \% Response } \\
\text { Rate }\end{array}$ \\
\hline Atkins et al., 1999 & IL-2 & 270 & 16 \\
\hline Legha, 1997 & IFN- $\alpha+$ IL-2 + chemo & 62 & 60 \\
\hline Itzhaki et al., 2011 & Adoptive cell transfer & 31 & 48 \\
\hline Prieto et al., 2012 & Ipilimumab + IL-2 & 36 & 25 \\
\hline Topalian et al., 2014 & Nivolumab & 107 & 31 \\
\hline Hamid et al., 2013 & Lambrolizumab & 135 & 38 \\
\hline Brahmer et al., 2012 & Anti-PD-L1 antibody & 55 & 17 \\
\hline
\end{tabular}

Chemo = chemotherapy; ipilimumab = anti-CTLA-4 antibody; lambrolizumab, nivolumab $=$ anti-PD-1 antibodies.

type BRAF. ${ }^{48}$ Therefore, targeted antibodies have been developed to inhibit the proliferation of BRAF V600E mutation-positive melanoma cells. Two of these antibodies, vemurafenib and dabrafenib, are FDA approved for the treatment of Stage IV metastatic melanoma. ${ }^{9}$ Vemurafenib obstructs the adenosine 5'-triphosphate-binding site of the active form of the BRAF kinase. ${ }^{8,87}$ Therefore, its effects are restricted to melanoma cells with BRAF V600E mutations that promote constitutive BRAF activation. At this time, BRAF inhibitors are the primary example of personalized medicine in melanoma immunotherapy; cytokinebased and checkpoint blockade-based therapy do not involve consideration of a patient's genetic profile. In later sections we outline a clinical multidisciplinary treatment approach to metastatic melanoma, which includes these immunotherapeutic advances.

\section{Cytokine-Based Therapy}

Cytokine-based therapy proves to be advantageous because it stimulates a potent immune response with a cytotoxic effect specific to metastatic cells.

\section{High-Dose IL-2 and Interferon}

Interleukin-2 is a cytokine that functions as a growth factor for T cells and natural killer (NK) cells. Administration of IL-2 can activate the Ras/MAPK and Jak/STAT pathways, both of which promote cytotoxic lymphocyte proliferation and differentiation. ${ }^{54}$ When given to melanoma patients with good performance status, IL-2 therapy elicited a response in $16 \%$ of patients, with $6 \%$ achieving a complete response. ${ }^{3}$

Interferons (IFNs) can be divided into 3 primary subtypes: IFN- $\alpha$, IFN- $\beta$, and IFN- $\gamma$. Therapy with IFN- $\beta$ is becoming an integral part of hepatitis $\mathrm{B}$ and multiple sclerosis therapy, ${ }^{12,62,80}$ and IFN- $\gamma$, an immune effector involved in Th1 response, has been shown to induce major histocompatibility complex (MHC) expression in malignant melanoma cells. ${ }^{66}$ However, IFN- $\alpha$, a cytokine that is produced in response to viral infection, is the primary focus of the majority of melanoma clinical trials. ${ }^{15,54}$ In addition to promoting apoptosis and inhibiting the growth of melanoma cells, $,, 15,35,76$ IFN- $\alpha$ stimulates the development of cytotoxic NK cells and CD8+ T cells. ${ }^{7}$ Treatment of metastatic melanoma with IFN- $\alpha$ alone resulted in a $15 \%$ response rate, which is comparable to data from IL-2 
studies. The response rate grew to $60 \%$ when IFN- $\alpha$ was administered in combination with chemotherapy.$^{45}$ As in IL-2, the potential for neurotoxicity may limit its use in metastatic melanomas with CNS involvement. ${ }^{39}$

Traditionally, administration of IL-2 has been discouraged for CNS metastases, due to heightened risk of increased intracranial pressure and neurotoxicity. ${ }^{51,64}$ The concern for neurotoxicity also applies for IFN treatment. ${ }^{39}$ However, recent studies of melanoma brain metastases suggest that these risks are not absolute. ${ }^{16,34,64}$ Additionally, these precautions prove less restrictive when treating extramedullary spinal metastases. Because these lesions impinge on but do not directly involve the spine, CNS penetration is a less relevant concern.

\section{Adoptive Cell Transfer}

The unique mechanism of adoptive cell transfer warrants special distinction from the previously mentioned forms of cytokine-based therapy. Adoptive cell transfer involves identification, ex vivo amplification, and readministration of tumor-infiltrating lymphocytes (TILs) to precipitate a robust cytokine response specific to metastatic melanoma. ${ }^{61}$ The readministration of TILs necessitates lymphodepletion through chemotherapy or wholebody radiation therapy. ${ }^{23,61,71}$ Administration of TILs in combination with IL-2 resulted in an overall response rate of 34\%-40\% and increased progression-free survival in patients with metastatic melanoma. ${ }^{36,71,90}$ These rates were similar in patients who were IL-2 naïve and in patients who had not responded to isolated IL-2 administration during previous therapy. ${ }^{71}$ Although this therapy showed some success, checkpoint blockade-based therapy and other targeted agents have demonstrated preferable tumor control and toxicity profiles.

\section{Checkpoint Blockade-Based Therapy}

Checkpoint blockade-based therapy targets pathways that promote self-tolerance and dampen immune response to prevent excessive inflammatory damage in surrounding tissue. ${ }^{58}$ Figure 1 depicts the fundamental steps in $\mathrm{T}$ cell-mediated immune response and illustrates the mechanisms of prominent checkpoint-based therapeutic mechanisms.

\section{Cytotoxic T-Lymphocyte Antigen-4 Blockade With Ipilimumab}

Efficient T-cell activation occurs in a 2-signal system. Signal 1 is the recognition of a foreign antigen presented in the frame of MHC-I by the T-cell receptor. Signal 2 , a costimulatory signal, involves the B7 ligand on the antigen-presenting cell binding to $\mathrm{CD} 28$, a costimulatory receptor expressed by naïve T cells. Cytotoxic T-lympho-

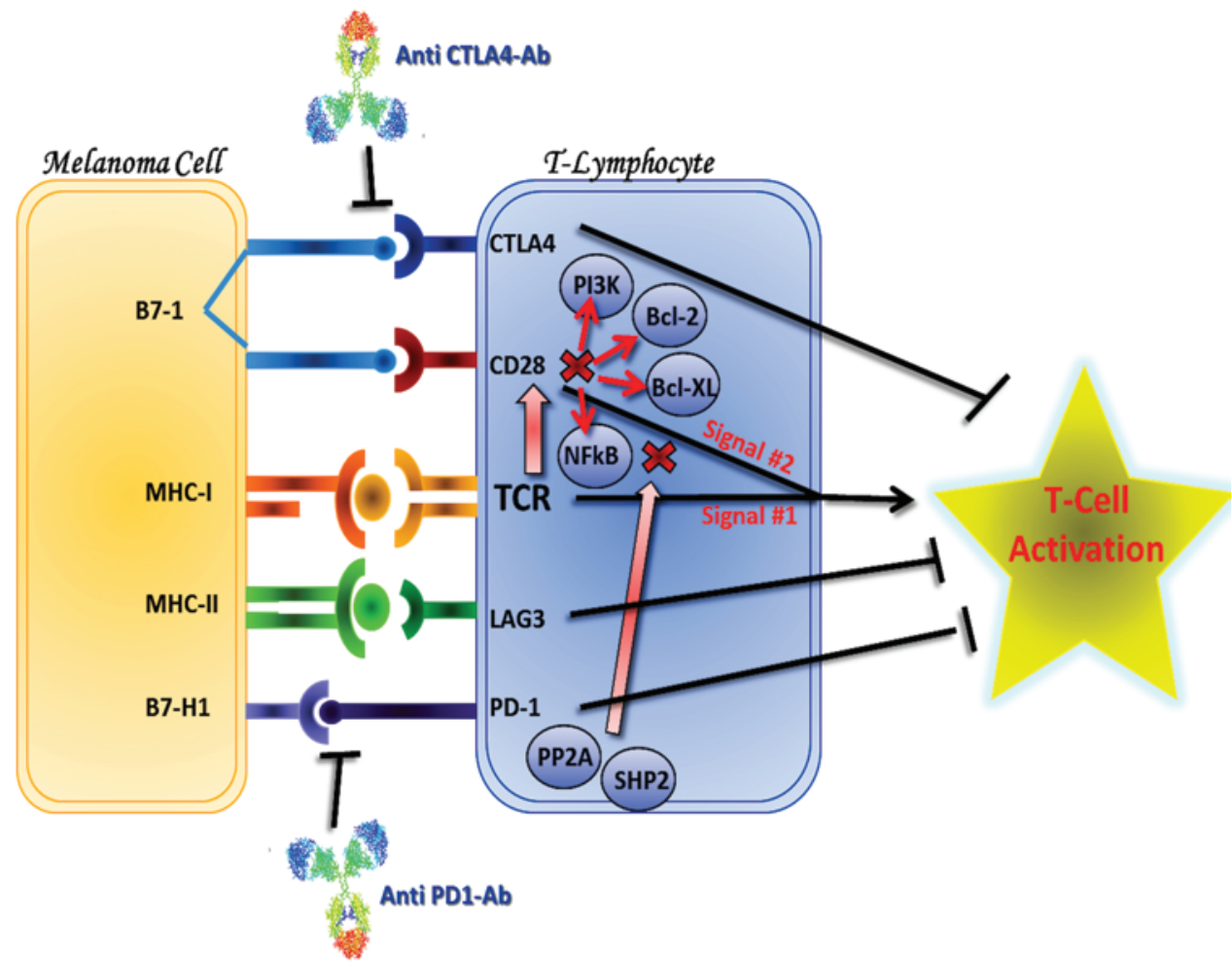

FIG. 1. Checkpoint blockade-based immunotherapy. The T-cell proliferation, differentiation, and cytokine release require 2 separate signal mechanisms. The Signal 1 involves T-cell receptor-mediated recognition of tumor antigen presented by MHC-I, which is located on the tumor cell. A second, costimulatory signal (Signal 2) involves B7 ligand, located on the tumor cell, binding to CD28, a receptor on the T cell. Both of these signals stimulate a variety of intracellular signaling pathways, which lead to upregulated activity of regulator proteins such as NF-KB, Bcl-2, and PI3K. These signals promote T-cell activation. However, other ligand-receptor binding pairs can inhibit these cascades and restrict T-cell activation. These inhibitory checkpoints include B7 binding to CTLA-4 and B7-H1 (PD-L1) binding to PD-1. Anti-CTLA-4 antibodies (ipilimumab) and anti-PD-1 antibodies facilitate T-cell activation by obstructing inhibitory checkpoint processes. $A b=$ antibody; TCR = T-cell receptor. 
cyte antigen-4 (CTLA-4) interrupts this costimulatory signal and restricts T-cell activation. ${ }^{22,46,58,59,69,74,75}$ Ipilimumab, a monoclonal antibody that targets CTLA-4, interrupts this inhibitory checkpoint process and facilitates Tcell activation. In patients with unresectable Stage III or IV melanoma, administration of ipilimumab resulted in a 47.2\% 1-year survival rate. ${ }^{56} \mathrm{~A}$ long-term follow-up of 177 patients with metastatic melanoma treated with ipilimumab revealed an 88-month median duration of objective response, which suggests that complete remission is possible in some patients. ${ }^{65}$ Additionally, Weber et al. demonstrated that ipilimumab is considered safe for patients with metastatic melanoma in whom there was CNS involvement. ${ }^{91}$

\section{Programmed Death-1/Programmed Death-1 Ligand 1 Blockade}

The programmed death-1 (PD-1) receptor is a T-cell receptor that negatively regulates immune response following interaction with certain ligands. Tumor ligands produced by melanoma cancer cells (programmed death -1 ligands 1 and 2 [PD-L1, PD-L2]) bind the PD-1 receptor and exploit this checkpoint. The PD-1/PD-L1 interaction inhibits T-cell proliferation, T-cell differentiation, and Th1-type cytokine release ${ }^{88}$ while increasing tumor resistance to cytotoxic T-cell activity. ${ }^{57}$ Therefore, disruption of the PD-1/PD-L1 interaction can promote cytokine production and enhance immune response.$^{84,85}$ Nivolumab and lambrolizumab are 2 examples of monoclonal antibodies that target PD-1. Nivolumab is associated with a $43 \%$ 2-year survival rate in metastatic melanoma, ${ }^{86}$ and lambrolizumab has demonstrated a response rate as high as $52 \%{ }^{31}$ in patients with metastatic melanoma. Monoclonal antibodies that target PD-L1 have also shown promising efficacy, with stable disease rates as high as $27 \%$ in patients with metastatic melanoma. ${ }^{10}$

\section{Open and Separation Surgery En Bloc Resection}

En bloc resection involves total excision of a tumor in 1 piece without violating the tumor margin and contaminating surrounding tissue..$^{17}$ The goal of this therapy is to achieve wide negative margins in the setting of solitary or oligometastatic disease, but the procedure carries a risk of significant morbidity. Surgeons may determine whether a patient qualifies for en bloc resection by using the Tomita score. Lower Tomita scores indicate a better prognosis and correspond with treatment strategies that promote long-term local control, such as en bloc resection. ${ }^{83}$ Additionally, clinicians may use the van der Linden score to determine a patient's prognosis. Lower van der Linden scores suggest a poorer prognosis, which precludes the rigors of en bloc resection in favor of a more palliative approach. ${ }^{89}$ Table 2 provides the methodology for calculating a patient's Tomita and van der Linden scores. However, considering the aggressive profile of metastatic melanoma and the risks of spinal cord and nerve root injury associated with aggressive surgery, en bloc resection of metastatic spinal melanoma is becoming a less favored intervention. ${ }^{17}$

\section{Separation Surgery}

In contrast to more invasive measures, "separation surgery" does not require total resection of the metastasis or the vertebral body, but relies on SRS to provide local tumor control..$^{50}$ The goal of separation surgery is to decompress epidural tumor from a posterolateral approach to circumferentially decompress the spinal cord and reconstitute the thecal sac, followed by long posterior segmental fixation (e.g., with pedicle screws) to provide immediate stability. ${ }^{43}$ Following surgery, patients undergo high-dose single-fraction or hypofractionated radiation to

\begin{tabular}{|c|c|c|}
\hline Scores & Tomita & van der Linden \\
\hline \multicolumn{3}{|l|}{ Score groupings } \\
\hline $0-3$ & Wide marginal excision for long-term control & 3 mos MOS \\
\hline $4-5$ & Marginal or intralesional excision for local control & 9 mos MOS \\
\hline $6-7$ & Palliative surgery for short-term palliation & \multirow[t]{2}{*}{18.7 mos MOS } \\
\hline $8-10$ & Nonop supportive care & \\
\hline \multicolumn{3}{|l|}{ Score determinants } \\
\hline \multirow[t]{4}{*}{ Primary tumor } & 1 = slow growth & $3=$ breast \\
\hline & 2 = moderate growth & $2=$ prostate \\
\hline & 4 = rapid growth & 1 = lung \\
\hline & & $0=$ other \\
\hline \multirow[t]{2}{*}{ Visceral metastases } & $2=$ treatable & $1=$ no \\
\hline & 4 = untreatable & $0=$ yes \\
\hline \multirow[t]{2}{*}{ Bone metastases (if present) } & 1 = solitary or isolated & \\
\hline & $2=$ multiple & \\
\hline \multirow[t]{3}{*}{ KPS } & & $2=$ KPS 80-100 \\
\hline & & $1=$ KPS $50-70$ \\
\hline & & $0=$ KPS $20-40$ \\
\hline
\end{tabular}

KPS = Karnofsky Performance Scale; MOS = median overall survival. 
ablate the residual gross and microscopic tumor volume. The radiation treatments spare normal tissue tolerance to at-risk structures, such as the spinal cord, since the separation surgery establishes an adequate distance for dose fall-off between the tumor margin and the spinal cord. ${ }^{50}$ Data show that spinal decompression surgery followed by cEBRT results in significantly better survival times and overall ambulation than cEBRT alone, with no significant difference in hospitalization time. ${ }^{60}$ However, the radioresistance of melanoma to cEBRT results in high local recurrence rates when used as a postoperative adjuvant. ${ }^{44}$

Laufer et al. demonstrated that local progression of spinal metastases was reduced in patients treated with separation surgery followed by high-dose single-fraction (24 Gy) or high-dose hypofractionated SRS (18-36 Gy in 3 fractions) compared with low-dose hypofractionated SRS (18-36 Gy in 5-6 fractions). ${ }^{43,44}$ All patients underwent separation surgery with the intent to establish sufficient distance between the residual tumor and the spinal cord, which facilitated the administration of radiosurgery. ${ }^{43,44}$ The 1-year recurrence rates were less than $10 \%$ in both high-dose cohorts. The responses are histology independent, indicating that even tumors that were radioresistant to cEBRT, such as melanoma, respond well to high-dose single-fraction or hypofractionated SRS. ${ }^{43,44}$ It is important to note that Laufer et al.'s study was not intended to assess the effects of separation surgery alone. Rather, it accents Patchell et al.'s observation that surgical decompression is beneficial for patients with paraspinal metastases. ${ }^{60}$

\section{Decision Making}

The NOMS criteria assist in determining whether patients qualify for separation surgery. The NOMS criteria incorporate evaluations of a patient's neurological deficits $(\mathrm{N})$, oncological features $(\mathrm{O})$, mechanical spinal instability $(\mathrm{M})$, and systemic disease progression (S) to clarify the ideal treatment plan. ${ }^{44}$ Neurological and oncological assessments include evaluation of the degree of epidural spinal cord compression and of functional deficits such as radiculopathy, and determination of the tumor's radiosensitivity ${ }^{44}$ If a neurological assessment reveals that epidural spinal cord compression is high grade, and if systemic evaluation reveals that the patient can tolerate surgery, then separation surgery is recommended prior to radiosurgery. ${ }^{44}$ For further clarification, Fig. 2 describes the management of a sample patient with metastatic melanoma who underwent separation surgery prior to SRS. Figure 3 provides an algorithm for a comprehensive multidisciplinary treatment approach.

\section{Stereotactic Radiosurgery}

The evolution of SRS underlies the utility of separation surgery and the decreasing reliance on aggressive resection. Stereotactic radiosurgery delivers single-fraction, high-dose radiation to a specific target. ${ }^{2}$ From a biological perspective, the efficacy of SRS derives from its ability to simultaneously kill individual tumor cells and damage tumor vasculature, both of which contribute to the release of proinflammatory cytokines that perpetuate an intense antitumor immune response. ${ }^{79}$ From a technical perspec-
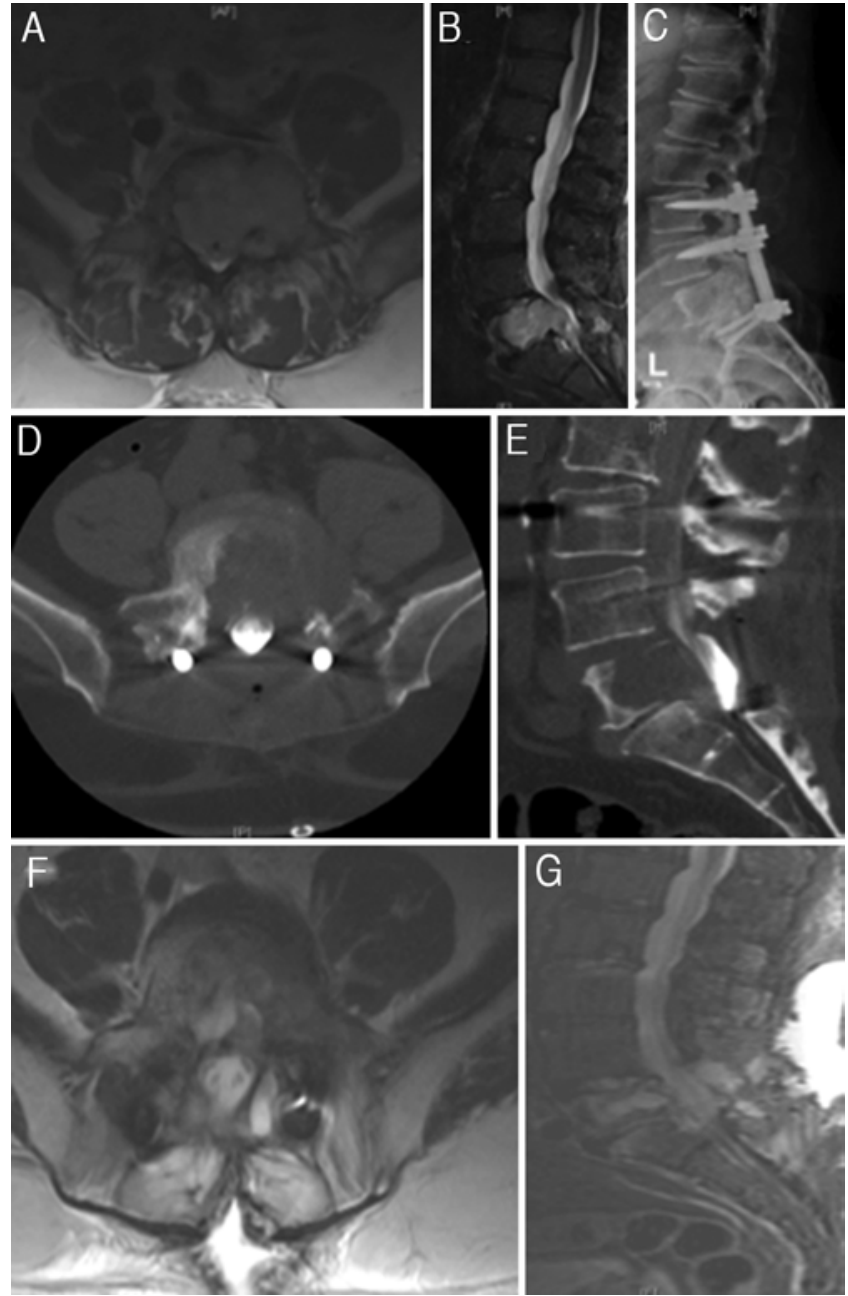

FIG. 2. Neuroimages obtained in a patient with metastatic melanoma treated with separation surgery. A 68-year-old man presented with severe mechanical radiculopathy. He had no history of cancer. Preoperative MRI studies showed a destructive lesion at L-5 causing high-grade thecal sac compression (A and B). The patient underwent separation surgery with a posterolateral resection of the epidural disease and L3-S1 pedicle screw fixation (C). He underwent a CT myelogram for radiation simulation and received a $24-G y$ single fraction (D and $E$ ). The patient had postoperative MRI studies showing patency of the spinal canal and maintained alignment (F and $\mathbf{G})$.

tive, the capacity of SRS to precisely limit target volumes facilitates steeper fall-off dose gradients than those of conventional radiation therapy. ${ }^{32}$ This implies that tissue in the immediate vicinity of the target volume is less likely to suffer radiation-induced damage.

Stereotactic radiosurgery can be performed with a variety of systems that operate with the following fundamental components: imaging and planning systems, a source of ionizing radiation, and a localization and placement procedure. ${ }^{1,11}$ Historically, the Gamma Knife was used to deliver SRS, but the Gamma Knife cannot reach spinal targets beyond C-2. Linear accelerator (LINAC) systems have since been adopted to deliver spinal radiosurgery. The LINAC produces high-energy x-rays as the radiation source and has fewer positional restrictions to aid more refined target selection. ${ }^{11}$ Most of today's systems do not 


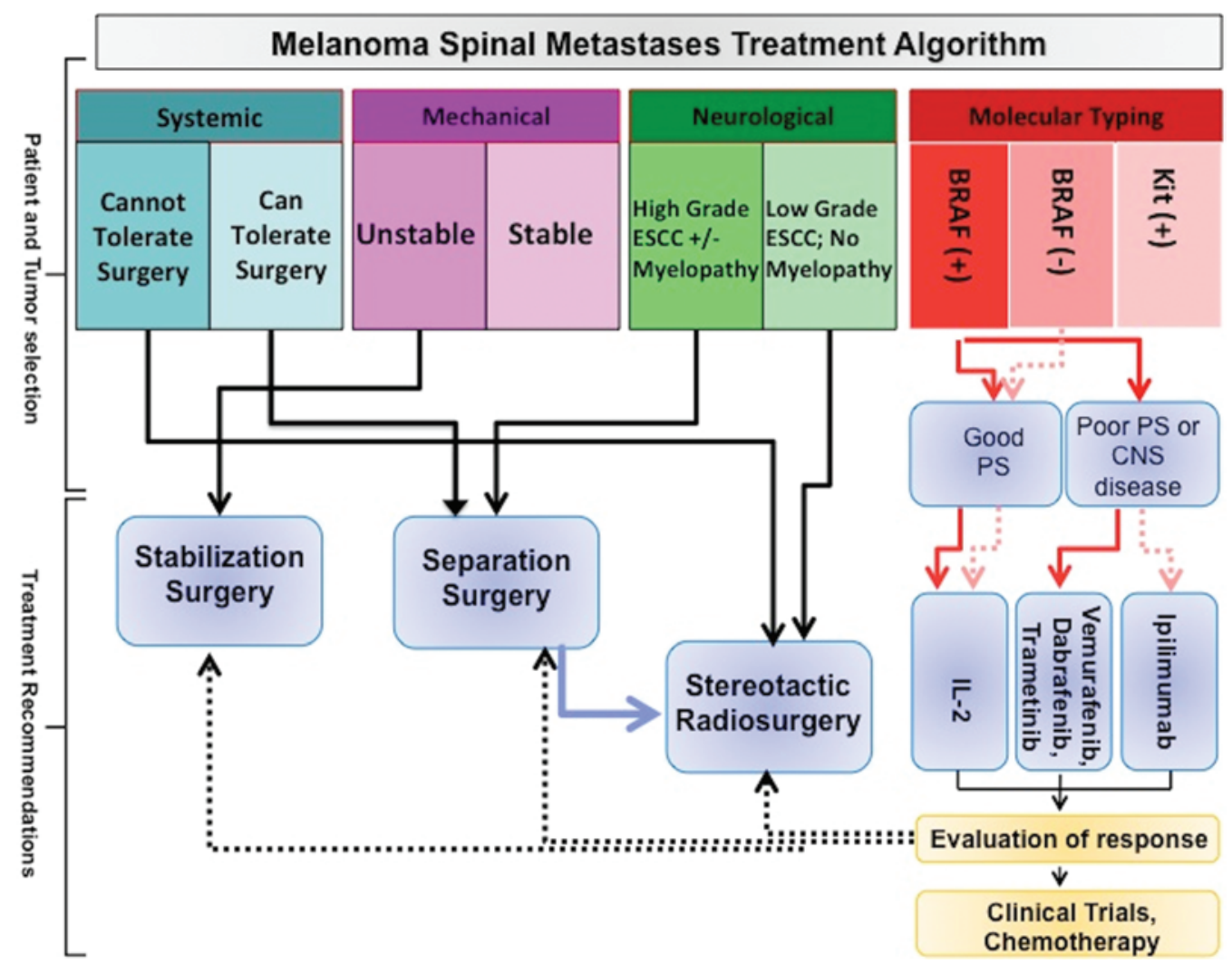

FIG. 3. Treatment algorithm for metastatic spinal melanoma based on the algorithms posited by Kaufman et al..$^{40}$ and Laufer et al. ${ }^{44}$ This schematic incorporates elements of the NOMS criteria ${ }^{44}$ with immunotherapeutic and molecular treatment schemes. Radiosurgery is necessary for treating extramedullary spinal melanoma metastases, because melanoma's radioresistant histology precludes cEBRT administration. If high-grade epidural spinal cord compression (ESCC) exists, decompressive separation surgery is required prior to radiosurgery. If patients present with mechanical instability, stabilization surgery is an independent surgical indication. Molecular typing of spinal melanoma metastases depends on whether the BRAF V600E mutation is present, because this influences the tumor's sensitivity to proteasome inhibitors and BRAF-kinase inhibitors, such as vemurafenib, dabrafenib, and trametinib. ${ }^{40}$ In the absence of a BRAF V600E mutation, CTLA-4 inhibitors such as ipilimumab, are indicated. If melanoma cells are Kit mutation-positive, they are less responsive to ipilimumab and nonresponsive to BRAF inhibitors. These patients are referred to clinical trials for more suitable immunotherapeutic regimens. PS = prognosis.

require frame fixation and can adjust for patient movement. ${ }^{1,11}$ Compared with cEBRT, SRS is associated with superior pain relief, long-term control, and survival in patients with spinal metastases. ${ }^{14,72}$ However, SRS can result in complications such as radiation-induced myelopathy, vertebral compression fractures, esophageal toxicity, and radiculopathy. ${ }^{72,73}$

\section{Data for Treatment of Metastatic Spinal Melanoma With SRS}

Although relatively few studies exist that specifically catalog the efficacy of SRS for treating metastatic spinal melanoma, the literature available reveals encouraging results. When malignant melanoma lesions were treated with CyberKnife radiosurgery (an image-guided and frameless LINAC system) and stereotactic body radiotherapy, local control was observed in $100 \%$ of patients with spinal lesions. ${ }^{37}$ Of these patients, $50 \%$ demonstrated complete response to therapy and $50 \%$ demonstrated a stable disease state. A study of separation surgery and SRS reported $100 \%$ local control in a set of 9 patients with metastatic spinal melanoma. ${ }^{43}$ Gerszten et al. also report $75 \%$ local radiographic control and $96 \%$ long-term pain improvement following CyberKnife SRS in a group of patients with 38 metastatic spinal melanoma lesions. ${ }^{27}$ Additionally, in a set of 9 patients with metastatic melanoma who were treated with the Elekta Synergy system (a frame-based LINAC system with 3D image guidance), $100 \%$ of patients with preoperative pain reported an improved or stable pain response at follow-up. ${ }^{30}$ Eight patients (89\%) demonstrated local control at last imaging follow-up. Two patients had undergone prior conventional radiation therapy, and $2 \mathrm{had}$ undergone prior single-fraction radiosurgery. No patients suffered from radiation-induced acute dermatitis, dysphagia, myelopathy, or fracture at the treated vertebral body. Additionally, no patients required subsequent SRS, and only 1 patient required subsequent decompression surgery.

In another study of 80 patients with metastatic spinal melanoma and renal cell carcinoma, Thiagaragan et al. reported an overall radiographic and symptomatic control rate of $92 \%$ following SRS. ${ }^{82}$ The patients were treated upfront with radiation doses ranging from 18 Gy to $24 \mathrm{~Gy}$, and melanoma lesions treated with 24 Gy demonstrated a $97 \%$ local control rate. Data regarding concurrent or prior immunotherapy and chemotherapy were not available for 
this cohort, but considering the recent advances of targeted immunotherapy, it is possible that these modalities may have provided additional advantage. These results demonstrate the promise of SRS in the treatment of metastatic spinal melanoma. The aforementioned studies are outlined in Table 3.

\section{Combination Therapy}

The benefits of immunotherapy, separation surgery, and SRS discussed earlier suggest that patients with metastatic spinal melanoma would benefit from individualized treatment regimens that combine these strategies. A study of 77 patients who underwent radiosurgery for melanoma brain metastases revealed that using ipilimumab as a supportive treatment was associated with a $27.5 \%$ increase in 2-year survival. ${ }^{41}$ However, a study of IFN therapy following SRS demonstrated no improvement in the outcomes of 39 patients with uveal melanoma. ${ }^{68}$ Finkelstein et al. revealed that 3-year overall survival and disease-free survival in patients with Stage III cutaneous melanoma were $48 \%$ and $43 \%$, respectively, following treatment with IFN and hypofractionated radiation therapy. ${ }^{25}$ No cases of acute Grade III myositis and only 9 cases of Grade III radiation dermatitis were reported in this study, contrasting with previous evidence of excessive toxicity associated with combination IFN and hypofractionated radiation. ${ }^{18,33,53}$ Additionally, a Phase I trial of IL-2 and stereotactic ablative body radiation therapy demonstrated an overall response rate of $62.5 \%$ in 7 patients with metastatic spinal melanoma. ${ }^{77}$

One of the benefits of combining ionizing radiation and immunotherapy is the induction of metastatic tumor regression at sites distant from the original radiation therapy location, a finding known as the abscopal effect. ${ }^{63}$ This effect was shown in a case report ${ }^{63}$ as well as in murine models, ${ }^{19-21}$ and it may be associated with hypofractionated rather than single-fraction radiation therapy. ${ }^{21} \mathrm{Ad}-$ ditionally, elevated concentrations of activated CD4 T cells and reduced concentrations of suppressor cells were observed after the administration of ipilimumab and radiation therapy, which suggests that radiation therapy may have enhanced the expansion of the patient's activated Tcell population and contributed to the systemic metastasis regression. ${ }^{63}$

TABLE 3. Studies of stereotactic radiosurgery for metastatic spinal melanoma

\begin{tabular}{lcccc}
\hline \multicolumn{1}{c}{ Authors \& Year } & $\begin{array}{c}\text { No. of } \\
\text { Patients }\end{array}$ & $\begin{array}{c}\text { Separation } \\
\text { Surgery }\end{array}$ & $\begin{array}{c}\text { \% Local } \\
\text { Control }\end{array}$ & $\begin{array}{c}\text { \% Pain } \\
\text { Relief }\end{array}$ \\
\hline Jahanshahi et al., 2012 & NR & No & 100 & NR \\
\hline Laufer et al., 2013 & 9 & Yes & 100 & NR \\
\hline Gerszten et al., 2007 & 38 & No & 75 & 96 \\
\hline Guckenberger et al., 2014 & 9 & No & 89 & $100^{*}$ \\
\hline Thiagaragan et al., 2010 & NR & No & $97 \dagger$ & NR \\
\hline
\end{tabular}

$\mathrm{NR}=$ not reported.

* This applies only to 7 patients. The other 2 patients did not exhibit preoperative pain.

† Applies only to patients treated with high-dose (24 Gy) stereotactic radiosurgery. The local control for all patients with metastatic melanoma was not reported.

\section{Future Directions}

Focused ultrasound uses high-intensity sound waves to destroy tumors with high temperatures. ${ }^{93}$ Following thermal ablation, the patient gradually reabsorbs the remaining tumor debris. Additionally, data show that focused ultrasound may function through immunomodulation to strengthen immune response to the remaining tumor cells via upregulation of Th1-type cytokine production and normalization of the CD4/CD8 T-cell ratio. ${ }^{24,52,70,93}$

The burgeoning success and popularity of immunotherapy and radiosurgery signify promising treatment options for patients with metastatic spinal melanoma. Additional research is necessary to determine the utility of combining SRS and immunotherapy to treat metastatic spinal melanoma. However, recent data suggest that cytokine-based therapies such as IFN and IL-2, or checkpoint blockadebased therapies such as ipilimumab, represent promising complements to separation surgery and SRS. Strategic use of combination therapy should improve local control and survival while lessening the risk of neurotoxicity and alleviating the need for onerous and costly total resections.

\section{Conclusions}

Metastatic melanoma to the spine is a significant challenge for clinicians. Immunotherapy coupled with separation surgery and SRS appears to offer a favorable outcome for many patients. However, further clinical studies are required to optimize the treatment approach for individual patients.

\section{References}

1. Adler JR Jr, Muacevic A, Romanelli P: CyberKnife radiosurgery, in Chin LS, Regine WF (eds): Principles and Practice of Stereotactic Radiosurgery. New York: Springer, 2008, pp 171-178

2. Ahmed KA, Stauder MC, Miller RC, Bauer HJ, Rose PS, Olivier KR, et al: Stereotactic body radiation therapy in spinal metastases. Int J Radiat Oncol Biol Phys 82:e803-e809, 2012

3. Atkins MB, Lotze MT, Dutcher JP, Fisher RI, Weiss G, Margolin K, et al: High-dose recombinant interleukin 2 therapy for patients with metastatic melanoma: analysis of 270 patients treated between 1985 and 1993. J Clin Oncol 17:2105-2116, 1999

4. Baker PK, Pettitt AR, Slupsky JR, Chen HJ, Glenn MA, Zuzel M, et al: Response of hairy cells to IFN-alpha involves induction of apoptosis through autocrine TNF-alpha and protection by adhesion. Blood 100:647-653, 2002

5. Balch CM, Buzaid AC, Soong SJ, Atkins MB, Cascinelli N, Coit DG, et al: Final version of the American Joint Committee on Cancer staging system for cutaneous melanoma. J Clin Oncol 19:3635-3648, 2001

6. Bedrosian I, Faries MB, Guerry D IV, Elenitsas R, Schuchter $\mathrm{L}$, Mick R, et al: Incidence of sentinel node metastasis in patients with thin primary melanoma $(<$ or $=1 \mathrm{~mm})$ with vertical growth phase. Ann Surg Oncol 7:262-267, 2000

7. Biron CA: Interferons alpha and beta as immune regulatorsa new look. Immunity 14:661-664, 2001

8. Bollag G, Hirth P, Tsai J, Zhang J, Ibrahim PN, Cho H, et al: Clinical efficacy of a RAF inhibitor needs broad target blockade in BRAF-mutant melanoma. Nature 467:596-599, 2010

9. Bollag G, Tsai J, Zhang J, Zhang C, Ibrahim P, Nolop K, et al: Vemurafenib: the first drug approved for BRAF-mutant cancer. Nat Rev Drug Discov 11:873-886, 2012 
10. Brahmer JR, Tykodi SS, Chow LQ, Hwu WJ, Topalian SL, Hwu P, et al: Safety and activity of anti-PD-L1 antibody in patients with advanced cancer. N Engl J Med 366:24552465, 2012

11. Bydon M, De la Garza-Ramos R, Bettagowda C, Gokaslan ZL, Sciubba DM: The use of stereotactic radiosurgery for the treatment of spinal axis tumors: a review. Clin Neurol Neurosurg 125:166-172, 2014

12. Carpi A, Nicolini A, Antonelli A, Ferrari P, Rossi G: Cytokines in the management of high risk or advanced breast cancer: an update and expectation. Curr Cancer Drug Targets 9:888-903, 2009

13. Cattell E, Kelly C, Middleton MR: Brain metastases in melanoma: a European perspective. Semin Oncol 29:513-517, 2002

14. Chan NK, Abdullah KG, Lubelski D, Steinmetz MP, Benzel EC, Shin JH, et al: Stereotactic radiosurgery for metastatic spine tumors. J Neurosurg Sci 58:37-44, 2014

15. Chin YE, Kitagawa M, Kuida K, Flavell RA, Fu XY: Activation of the STAT signaling pathway can cause expression of caspase 1 and apoptosis. Mol Cell Biol 17:5328-5337, 1997

16. Chu MB, Fesler MJ, Armbrecht ES, Fosko SW, Hsueh E, Richart JM: High-Dose Interleukin-2 (HD IL-2) therapy should be considered for treatment of patients with melanoma brain metastases. Chemother Res Pract 2013:726925, 2013

17. Cloyd JM, Acosta FL Jr, Polley MY, Ames CP: En bloc resection for primary and metastatic tumors of the spine: a systematic review of the literature. Neurosurgery 67:435-445, 2010

18. Conill C, Jorcano S, Domingo-Domènech J, Marruecos J, Vilella R, Malvehy J, et al: Toxicity of combined treatment of adjuvant irradiation and interferon alpha2b in high-risk melanoma patients. Melanoma Res 17:304-309, 2007

19. Demaria S, Bhardwaj N, McBride WH, Formenti SC: Combining radiotherapy and immunotherapy: a revived partnership. Int J Radiat Oncol Biol Phys 63:655-666, 2005

20. Demaria S, Kawashima N, Yang AM, Devitt ML, Babb JS, Allison JP, et al: Immune-mediated inhibition of metastases after treatment with local radiation and CTLA- 4 blockade in a mouse model of breast cancer. Clin Cancer Res 11:728734,2005

21. Dewan MZ, Galloway AE, Kawashima N, Dewyngaert JK, Babb JS, Formenti SC, et al: Fractionated but not single-dose radiotherapy induces an immune-mediated abscopal effect when combined with anti-CTLA-4 antibody. Clin Cancer Res 15:5379-5388, 2009

22. Egen JG, Allison JP: Cytotoxic T lymphocyte antigen-4 accumulation in the immunological synapse is regulated by TCR signal strength. Immunity 16:23-35, 2002

23. Ellebaek E, Iversen TZ, Junker N, Donia M, Engell-Noerregaard L, Met Ö, et al: Adoptive cell therapy with autologous tumor infiltrating lymphocytes and low-dose Interleukin-2 in metastatic melanoma patients. J Transl Med 10:169, 2012

24. Evrard S, Menetrier-Caux C, Biota C, Neaud V, MathoulinPélissier S, Blay JY, et al: Cytokines pattern after surgical radiofrequency ablation of liver colorectal metastases. Gastroenterol Clin Biol 31:141-145, 2007

25. Finkelstein SE, Trotti A, Rao N, Reintgen D, Cruse W, Feun L, et al: The Florida Melanoma Trial I: a prospective multicenter phase I/II trial of postoperative hypofractionated adjuvant radiotherapy with concurrent interferon-Alfa- $2 \mathrm{~b}$ in the treatment of advanced stage III melanoma with long-term toxicity follow-up. ISRN Immunology 2012:324235, 2012

26. Garbe C, Eigentler TK, Keilholz U, Hauschild A, Kirkwood JM: Systematic review of medical treatment in melanoma: current status and future prospects. Oncologist 16:5-24, 2011

27. Gerszten PC, Burton SA, Ozhasoglu C, Welch WC: Ra- diosurgery for spinal metastases: clinical experience in 500 cases from a single institution. Spine (Phila Pa 1976) 32:193-199, 2007

28. Gerszten PC, Burton SA, Quinn AE, Agarwala SS, Kirkwood JM: Radiosurgery for the treatment of spinal melanoma metastases. Stereotact Funct Neurosurg 83:213-221, 2005

29. Gokaslan ZL, Aladag MA, Ellerhorst JA: Melanoma metastatic to the spine: a review of 133 cases. Melanoma Res 10:78-80, 2000

30. Guckenberger M, Mantel F, Gerszten PC, Flickinger JC, Sahgal A, Létourneau D, et al: Safety and efficacy of stereotactic body radiotherapy as primary treatment for vertebral metastases: a multi-institutional analysis. Radiat Oncol 9:226, 2014

31. Hamid O, Robert C, Daud A, Hodi FS, Hwu WJ, Kefford R, et al: Safety and tumor responses with lambrolizumab (antiPD-1) in melanoma. N Engl J Med 369:134-144, 2013

32. Harel R, Zach L: Spine radiosurgery for spinal metastases: indications, technique and outcome. Neurol Res 36:550-556, 2014

33. Hazard LJ, Sause WT, Noyes RD: Combined adjuvant radiation and interferon-alpha $2 \mathrm{~B}$ therapy in high-risk melanoma patients: the potential for increased radiation toxicity. Int J Radiat Oncol Biol Phys 52:796-800, 2002

34. Hodi FS, Oble DA, Drappatz J, Velazquez EF, Ramaiya N, Ramakrishna N, et al: CTLA-4 blockade with ipilimumab induces significant clinical benefit in a female with melanoma metastases to the CNS. Nat Clin Pract Oncol 5:557-561, 2008

35. Huang S, Bucana CD, Van Arsdall M, Fidler IJ: Stat1 negatively regulates angiogenesis, tumorigenicity and metastasis of tumor cells. Oncogene 21:2504-2512, 2002

36. Itzhaki O, Hovav E, Ziporen Y, Levy D, Kubi A, Zikich D, et al: Establishment and large-scale expansion of minimally cultured "young" tumor infiltrating lymphocytes for adoptive transfer therapy. J Immunother 34:212-220, 2011

37. Jahanshahi P, Nasr N, Unger K, Batouli A, Gagnon GJ: Malignant melanoma and radiotherapy: past myths, excellent local control in 146 studied lesions at Georgetown University, and improving future management. Front Oncol 2:167, 2012

38. Jemal A, Siegel R, Xu J, Ward E: Cancer statistics, 2010. CA Cancer J Clin 60:277-300, 2010

39. Jonasch E, Kumar UN, Linette GP, Hodi FS, Soiffer RJ, Ryan $\mathrm{BF}$, et al: Adjuvant high-dose interferon alfa- $2 \mathrm{~b}$ in patients with high-risk melanoma. Cancer J 6:139-145, 2000

40. Kaufman HL, Kirkwood JM, Hodi FS, Agarwala S, Amatruda T, Bines SD, et al: The Society for Immunotherapy of Cancer consensus statement on tumour immunotherapy for the treatment of cutaneous melanoma. Nat Rev Clin Oncol 10:588-598, 2013

41. Knisely JP, Yu JB, Flanigan J, Sznol M, Kluger HM, Chiang VL: Radiosurgery for melanoma brain metastases in the ipilimumab era and the possibility of longer survival. J Neurosurg 117:227-233, 2012

42. Ku A, Henry A, Tunkel R, Lachmann E, Nagler W: Lumbosacral radiculopathy secondary to L5 metastatic melanoma of unknown primary. Arch Phys Med Rehabil 77:307-309, 1996

43. Laufer I, Iorgulescu JB, Chapman T, Lis E, Shi W, Zhang Z, et al: Local disease control for spinal metastases following "separation surgery" and adjuvant hypofractionated or highdose single-fraction stereotactic radiosurgery: outcome analysis in 186 patients. J Neurosurg Spine 18:207-214, 2013

44. Laufer I, Rubin DG, Lis E, Cox BW, Stubblefield MD, Yamada Y, et al: The NOMS framework: approach to the treatment of spinal metastatic tumors. Oncologist 18:744-751, 2013

45. Legha SS: Durable complete responses in metastatic melanoma treated with interleukin-2 in combination with interferon alpha and chemotherapy. Semin Oncol 24 (1 Suppl 4):S39S43, 1997 
46. Linsley PS, Greene JL, Brady W, Bajorath J, Ledbetter JA, Peach R: Human B7-1 (CD80) and B7-2 (CD86) bind with similar avidities but distinct kinetics to CD28 and CTLA-4 receptors. Immunity 1:793-801, 1994

47. Lippitz B, Lindquist C, Paddick I, Peterson D, O’Neill K, Beaney R: Stereotactic radiosurgery in the treatment of brain metastases: the current evidence. Cancer Treat Rev 40:4859,2014

48. Long GV, Trefzer U, Davies MA, Kefford RF, Ascierto PA, Chapman PB, et al: Dabrafenib in patients with Val600Glu or Val600Lys BRAF-mutant melanoma metastatic to the brain (BREAK-MB): a multicentre, open-label, phase 2 trial. Lancet Oncol 13:1087-1095, 2012

49. McWilliams RR, Rao RD, Buckner JC, Link MJ, Markovic S, Brown PD: Melanoma-induced brain metastases. Expert Rev Anticancer Ther 8:743-755, 2008

50. Moussazadeh N, Laufer I, Yamada Y, Bilsky MH: Separation surgery for spinal metastases: effect of spinal radiosurgery on surgical treatment goals. Cancer Contr 21:168-174, 2014

51. Myint AM, Schwarz MJ, Steinbusch HW, Leonard BE: Neuropsychiatric disorders related to interferon and interleukins treatment. Metab Brain Dis 24:55-68, 2009

52. Napoletano C, Taurino F, Biffoni M, De Majo A, Coscarella $\mathrm{G}$, Bellati F, et al: RFA strongly modulates the immune system and anti-tumor immune responses in metastatic liver patients. Int J Oncol 32:481-490, 2008

53. Nguyen NP, Levinson B, Dutta S, Karlsson U, Alfieri A, Childress $\mathrm{C}$, et al: Concurrent interferon-alpha and radiation for head and neck melanoma. Melanoma Res 13:67-71, 2003

54. Nicholas C, Lesinski GB: Immunomodulatory cytokines as therapeutic agents for melanoma. Immunotherapy 3:673690, 2011

55. O'Connell MP, Marchbank K, Webster MR, Valiga AA, Kaur A, Vultur A, et al: Hypoxia induces phenotypic plasticity and therapy resistance in melanoma via the tyrosine kinase receptors ROR1 and ROR2. Cancer Discov 3:1378-1393, 2013

56. O'Day SJ, Maio M, Chiarion-Sileni V, Gajewski TF, Pehamberger H, Bondarenko IN, et al: Efficacy and safety of ipilimumab monotherapy in patients with pretreated advanced melanoma: a multicenter single-arm phase II study. Ann Oncol 21:1712-1717, 2010

57. Okazaki T, Honjo T: The PD-1-PD-L pathway in immunological tolerance. Trends Immunol 27:195-201, 2006

58. Pardoll DM: The blockade of immune checkpoints in cancer immunotherapy. Nat Rev Cancer 12:252-264, 2012

59. Parry RV, Chemnitz JM, Frauwirth KA, Lanfranco AR, Braunstein I, Kobayashi SV, et al: CTLA-4 and PD-1 receptors inhibit T-cell activation by distinct mechanisms. Mol Cell Biol 25:9543-9553, 2005

60. Patchell RA, Tibbs PA, Regine WF, Payne R, Saris S, Kryscio RJ, et al: Direct decompressive surgical resection in the treatment of spinal cord compression caused by metastatic cancer: a randomised trial. Lancet 366:643-648, 2005

61. Phan GQ, Rosenberg SA: Adoptive cell transfer for patients with metastatic melanoma: the potential and promise of cancer immunotherapy. Cancer Contr 20:289-297, 2013

62. Plosker GL: Interferon- $\beta$-1b: a review of its use in multiple sclerosis. CNS Drugs 25:67-88, 2011

63. Postow MA, Callahan MK, Barker CA, Yamada Y, Yuan J, Kitano S, et al: Immunologic correlates of the abscopal effect in a patient with melanoma. N Engl J Med 366:925-931, 2012

64. Powell S, Dudek AZ: Single-institution outcome of high-dose interleukin-2 (HD IL-2) therapy for metastatic melanoma and analysis of favorable response in brain metastases. Anticancer Res 29:4189-4193, 2009

65. Prieto PA, Yang JC, Sherry RM, Hughes MS, Kammula US, White DE, et al: CTLA-4 blockade with ipilimumab: long- term follow-up of 177 patients with metastatic melanoma. Clin Cancer Res 18:2039-2047, 2012

66. Propper DJ, Chao D, Braybrooke JP, Bahl P, Thavasu P, Balkwill F, et al: Low-dose IFN-gamma induces tumor MHC expression in metastatic malignant melanoma. Clin Cancer Res 9:84-92, 2003

67. Reed KB, Brewer JD, Lohse CM, Bringe KE, Pruitt CN, Gibson LE: Increasing incidence of melanoma among young adults: an epidemiological study in Olmsted County, Minnesota. Mayo Clin Proc 87:328-334, 2012

68. Richtig E, Langmann G, Schlemmer G, Müllner K, Papaefthymiou G, Bergthaler P, et al: [Safety and efficacy of interferon alfa- $2 b$ in the adjuvant treatment of uveal melanoma.] Ophthalmologe 103:506-511, 2006 (Ger)

69. Riley JL, Mao M, Kobayashi S, Biery M, Burchard J, Cavet $\mathrm{G}$, et al: Modulation of TCR-induced transcriptional profiles by ligation of CD28, ICOS, and CTLA-4 receptors. Proc Natl Acad Sci U S A 99:11790-11795, 2002

70. Rosberger DF, Coleman DJ, Silverman R, Woods S, Rondeau $\mathrm{M}$, Cunningham-Rundles S: Immunomodulation in choroidal melanoma: reversal of inverted CD4/CD8 ratios following treatment with ultrasonic hyperthermia. Biotechnol Ther 5:59-68, 1994

71. Rosenberg SA, Yannelli JR, Yang JC, Topalian SL, Schwartzentruber DJ, Weber JS, et al: Treatment of patients with metastatic melanoma with autologous tumor-infiltrating lymphocytes and interleukin 2. J Natl Cancer Inst 86:1159-1166, 1994

72. Sahgal A, Larson DA, Chang EL: Stereotactic body radiosurgery for spinal metastases: a critical review. Int J Radiat Oncol Biol Phys 71:652-665, 2008

73. Sahgal A, Whyne CM, Ma L, Larson DA, Fehlings MG: Vertebral compression fracture after stereotactic body radiotherapy for spinal metastases. Lancet Oncol 14:e310-e320, 2013

74. Schneider H, Downey J, Smith A, Zinselmeyer BH, Rush C, Brewer JM, et al: Reversal of the TCR stop signal by CTLA4. Science 313:1972-1975, 2006

75. Schneider H, Mandelbrot DA, Greenwald RJ, Ng F, Lechler $\mathrm{R}$, Sharpe AH, et al: Cutting edge: CTLA-4 (CD152) differentially regulates mitogen-activated protein kinases (extracellular signal-regulated kinase and c-Jun N-terminal kinase) in CD4+ T cells from receptor/ligand-deficient mice. J Immunol 169:3475-3479, 2002

76. Selleri C, Sato T, Del Vecchio L, Luciano L, Barrett AJ, Rotoli B, et al: Involvement of Fas-mediated apoptosis in the inhibitory effects of interferon-alpha in chronic myelogenous leukemia. Blood 89:957-964, 1997

77. Seung SK, Curti BD, Crittenden M, Walker E, Coffey T, Siebert JC, et al: Phase 1 study of stereotactic body radiotherapy and interleukin-2-tumor and immunological responses. SciTransl Med 4:137ra174, 2012

78. Shah DJ, Dronca RS: Latest advances in chemotherapeutic, targeted, and immune approaches in the treatment of metastatic melanoma. Mayo Clin Proc 89:504-519, 2014

79. Song CW, Kim MS, Cho LC, Dusenbery K, Sperduto PW: Radiobiological basis of SBRT and SRS. Int J Clin Oncol 19:570-578, 2014

80. Sonneveld MJ, Rijckborst V, Boucher CA, Hansen BE, Janssen HL: Prediction of sustained response to peginterferon alfa-2b for hepatitis B e antigen-positive chronic hepatitis B using on-treatment hepatitis B surface antigen decline. Hepatology 52:1251-1257, 2010

81. Stewart WR, Gelberman RH, Harrelson JM, Seigler HF: Skeletal metastases of melanoma. J Bone Joint Surg Am 60:645-649, 1978

82. Thiagaragan A, Yamada Y, Lovelock DM, Cox BW, Zelefsky MJ, Bilsky M: Stereotactic radiosurgery: a new paradigm for melanoma and renal cell carcinoma spine metastases. J Clin Oncol 28 (Suppl):2030, 2010 (Abstract) 
83. Tomita K, Kawahara N, Kobayashi T, Yoshida A, Murakami H, Akamaru T: Surgical strategy for spinal metastases. Spine (Phila Pa 1976) 26:298-306, 2001

84. Topalian SL, Drake CG, Pardoll DM: Targeting the PD-1/B7H1(PD-L1) pathway to activate anti-tumor immunity. Curr Opin Immunol 24:207-212, 2012

85. Topalian SL, Hodi FS, Brahmer JR, Gettinger SN, Smith DC, McDermott DF, et al: Safety, activity, and immune correlates of anti-PD-1 antibody in cancer. N Engl J Med 366:24432454,2012

86. Topalian SL, Sznol M, McDermott DF, Kluger HM, Carvajal RD, Sharfman WH, et al: Survival, durable tumor remission, and long-term safety in patients with advanced melanoma receiving nivolumab. J Clin Oncol 32:1020-1030, 2014

87. Tsai J, Lee JT, Wang W, Zhang J, Cho H, Mamo S, et al: Discovery of a selective inhibitor of oncogenic B-Raf kinase with potent antimelanoma activity. Proc Natl Acad Sci U S A 105:3041-3046, 2008

88. Tseng SY, Otsuji M, Gorski K, Huang X, Slansky JE, Pai SI, et al: B7-DC, a new dendritic cell molecule with potent costimulatory properties for T cells. J Exp Med 193:839-846, 2001

89. van der Linden YM, Dijkstra SP, Vonk EJ, Marijnen CA, Leer JW: Prediction of survival in patients with metastases in the spinal column: results based on a randomized trial of radiotherapy. Cancer 103:320-328, 2005

90. Weber J, Atkins M, Hwu P, Radvanyi L, Sznol M, Yee C: White paper on adoptive cell therapy for cancer with tumorinfiltrating lymphocytes: a report of the CTEP subcommittee on adoptive cell therapy. Clin Cancer Res 17:1664-1673, 2011
91. Weber JS, Amin A, Minor D, Siegel J, Berman D, O'Day SJ: Safety and clinical activity of ipilimumab in melanoma patients with brain metastases: retrospective analysis of data from a phase 2 trial. Melanoma Res 21:530-534, 2011

92. Wouters J, Stas M, Gremeaux L, Govaere O, Van den Broeck A, Maes H, et al: The human melanoma side population displays molecular and functional characteristics of enriched chemoresistance and tumorigenesis. PLoS ONE 8: 76550 , 2013

93. Wu F: High intensity focused ultrasound ablation and antitumor immune response. J Acoust Soc Am 134:1695-1701, 2013

94. Yamada Y, Bilsky MH, Lovelock DM, Venkatraman ES, Toner S, Johnson J, et al: High-dose, single-fraction imageguided intensity-modulated radiotherapy for metastatic spinal lesions. Int J Radiat Oncol Biol Phys 71:484-490, 2008

\section{Author Contributions}

Conception and design: Sheehan, Caruso, Cohen-Inbar. Acquisition of data: Caruso. Analysis and interpretation of data: Caruso, Cohen-Inbar. Drafting the article: Caruso. Critically revising the article: all authors. Reviewed submitted version of manuscript: all authors. Approved the final version of the manuscript on behalf of all authors: Sheehan. Administrative/technical/material support: Bilsky, Gerszten. Study supervision: Sheehan, Cohen-Inbar.

\section{Correspondence}

Jason P. Sheehan, Department of Neurological Surgery, University of Virginia Health System, Box 800212, Charlottesville, VA 22908.email: jsheehan@virginia.edu. 